Cahiers $d u$ MONDE RUSSE

\section{Cahiers du monde russe}

Russie - Empire russe - Union soviétique et États indépendants

$49 / 4 \mid 2008$

Destins individuels et terreur. Jeunesse dans la société post-stalinienne

\title{
Jeffrey J. Rossman, Worker Resistance under Stalin
}

\section{Gábor T. Rittersporn}

\section{(2) OpenEdition}

Journals

Édition électronique

URL : https://journals.openedition.org/monderusse/6940

DOI : 10.4000/monderusse. 6940

ISSN : $1777-5388$

Éditeur

Éditions de l'EHESS

Édition imprimée

Date de publication : 28 décembre 2008

Pagination : 743-748

ISBN : 978-2-7132-2197-2

ISSN : $1252-6576$

Référence électronique

Gábor T. Rittersporn, « Jeffrey J. Rossman, Worker Resistance under Stalin », Cahiers du monde russe [En ligne], 49/4 | 2008, mis en ligne le 23 décembre 2009, consulté le 03 septembre 2022. URL: http://journals.openedition.org/monderusse/6940; DOI : https://doi.org/10.4000/monderusse.6940

Ce document a été généré automatiquement le 3 septembre 2022

Tous droits réservés 


\title{
Jeffrey J. Rossman, Worker Resistance under Stalin
}

\author{
Gábor T. Rittersporn
}

\section{RÉFÉRENCE}

Jeffrey J. ROSSMAN, Worker Resistance under Stalin. Class and Revolution on the Shop Floor. Cambridge, MA : Harvard University Press, 2007, 314 p.

1 La nouvelle orthodoxie de la recherche sur l'histoire soviétique évite soigneusement de parler de résistance au régime stalinien. Résistance, dit-on, implique une personnalité autonome par rapport au régime. Or les citoyens de l'URSS auraient été incapables de garder une distance par rapport aux normes fixées par l'État. Il leur manquait un point d'appui au-delà du système. Le concept de résistance est un piège pour le chercheur, car il suppose l'hypothèse improbable d'un sujet capable d'intérioriser des valeurs libérales ${ }^{1}$.

2 Que dire dès lors du livre de Jeffrey J. Rossman, qui ose explorer la résistance au régime stalinien et, plus encore, la résistance ouvrière? Comment les travailleurs pouvaient-ils tenir tête aux bolcheviks en étant indiscutablement influencés par le discours officiel qui les célébrait comme la classe dirigeante?

3 Dans un certain nombre de centres industriels, le printemps de 1932 fut marqué par une forte agitation qui se transforma par endroits en révoltes. Ce fut le cas de la région industrielle d'Ivanovo. Les évènements sont désormais bien documentés. Une première mobilisation avait déjà eu lieu en 1928 contre la politique du gouvernement. Pourtant, les travailleurs de la ville et des environs avaient activement participé à la Révolution. Qu'ils aient été bolcheviks ou non, leur soulèvement fut décisif pour renverser l'ancien régime dans la province. Dans les années 1920, le parti comptait relativement peu de membres parmi les ouvriers d'Ivanovo, mais la sympathie des masses lui était acquise. Celles-ci bénéficièrent de la NEP qui succéda aux années difficiles de la guerre civile.

4 La région était un bastion du textile, produisait un tiers du tissu fabriqué en URSS et employait plus de $12 \%$ des ouvriers de l'industrie soviétique. Elle occupait la troisième 
place dans les régions industrielles quant à la production de textile et la concentration de main-d'œuvre, juste après Moscou et Leningrad. Tout ce qui s'y passait revêtait de ce fait une grande importance pour le régime.

5 Les ouvriers en étaient conscients et savaient tempérer le zèle du gouvernement lorsque celui-ci décidait d'intensifier la production. Un ralentissement du travail et de courtes grèves parvenaient à freiner les campagnes tayloristes. Mais la situation se détériora avec l'industrialisation. L'État-parti projeta de diminuer d'un tiers les investissements et d'accroître la production du textile de plus de $85 \%$ en n'augmentant la main-d'œuvre que de quelque $12 \%$. L'exigence d'une productivité renforcée était rendue plus intolérable par la dévalorisation des revenus et la pénurie qu'entretenait la collectivisation de l'agriculture.

6 Les ouvriers les plus qualifiés en furent les premières victimes. Leur charge de travail, allant jusqu'à doubler, était devenue insupportable. Mais tout le monde était touché. La détérioration dramatique des conditions de vie et l'abîme qui séparait les promesses du régime de l'expérience vécue étaient flagrants. La situation devint explosive vers l'automne 1928. L'année fut marquée par des grèves sporadiques qui se transformèrent en de larges arrêts de travail et des assemblées orageuses où les délégués des ouvriers s'en prirent au régime et revendiquèrent une amélioration de l'approvisionnement et des conditions de travail. Le Comité central finit par augmenter les normes de rationnement dans les centres urbains.

7 Les conflits de 1932 furent précédés par de nouvelles vagues d'intensification de la production, une réduction des salaires, l'aggravation de la pénurie - et une remarquable radicalisation des masses. Rossman décrit la misère à laquelle les ouvriers d'Ivanovo étaient confrontés. Dans deux villes, occupations d'usines, démonstrations et assemblées extraordinaires mobilisèrent plusieurs milliers d'ouvriers. Ainsi, à Teikovo, une tentative de marche de la faim sur la capitale de la région se solda par un échec. À Vichuga, l'arrestation d'un leader du mouvement et un assaut de la police provoquèrent de véritables émeutes. Le parti dépêcha sur place Lazar Kaganovič pour rameuter les bolcheviks désarçonnés et rétablir l'ordre.

8 Il serait exagéré de surestimer la portée des affrontements d'Ivanovo, même si des conflits similaires se produisirent ailleurs dans le pays. Pourquoi faire si grand cas de la révolte de quelques milliers d'ouvriers? Peut-on vraiment prétendre qu'ils résistèrent au régime? L'auteur montre qu'ils invoquèrent la Révolution, le pouvoir des soviets et les droits hypothétiques accordés aux ouvriers. Il semble donc que leur projet ait été tributaire du discours officiel. Le rôle particulier de l'industrie textile et l'importance d'Ivanovo dans la branche ont sans doute inquiété les autorités, mais le régime a-t-il vraiment fait face à un défi majeur?

9 Ce n'est pas ce que prétend Rossman. Il se demande seulement si la distance manifeste qui séparait les attentes des rebelles de la pratique bolchevique a suffi à les inciter à une opposition radicale, et si leur contestation était synonyme de résistance à l'État-parti. La réponse n'est pas simple si l'on se place dans la perspective des nouvelles hypothèses sur le concept de résistance.

10 L'auteur montre que les contestataires avaient un programme incompatible avec celui du régime. La Révolution avait renforcé l'attachement des masses à une forme d'économie morale, à une vision populaire de la justice sociale. Celle-ci était fondée sur l'utopie égalitaire d'une communauté qui serait à même de subvenir aux besoins de ses 
membres et à garantir leur dignité. Néanmoins, ces masses avaient tendance à confondre les communautés où elles vivaientavec cette utopie.

11 Puisque le nouveau régime célébrait les ouvriers comme la classe dominante, il était logique de leur part de parler au nom du prolétariat victorieux et d'exiger que l'Étatparti réalise l'utopie qu'ils avaient quelque raison de projeter dans le discours bolchevik. Or, la politique des dirigeants allait manifestement à l'encontre de ce qu'ils attendaient. Pas besoin d'être un fin savant des sciences sociales pour s'en rendre compte : il suffisait de ne plus pouvoir joindre les deux bouts et d'entendre ses enfants réclamer du pain.

La Révolution et les soviets dont les masses se réclamaient n'étaient pas vraiment ceux des bolcheviks. Ils se trouvaient au-delà de l'univers quotidien du travailleur. On est obligé d'admettre que les rebelles d'Ivanovo n'étaient pas des libéraux délicats. Ils préféraient vivre dans des familles patriarcales et des communautés passablement autoritaires, se souciant moins de l'émancipation des femmes qu'on ne pourrait l'attendre de champions de l'égalitarisme. Les ouvriers étaient même prêts à recourir à la violence. Il n'en reste pas moins que le monde dans lequel ils vivaient n'était pas celui que les bolcheviks promettaient et que nombre de travailleurs n'étaient pas dupes des lendemains que l'État-parti était en train de construire.Le champ de bataille que le régime préparait pour les travailleurs de choc n'était certainement pas la terre promise de la vie communautaire. Les contraintes que l'État-parti imposait au nom d'un avenir incertain n'était aucunement la liberté inconditionnelle du rêve plébéien.

C'était bien pour obtenir gain de cause que les révoltés d'Ivanovo s'adressaient au gouvernement. Dans leurs assemblées, ils discutaient des requêtes qu'ils allaient présenter et n'étaient pas là pour échafauder de nouveaux modèles sociopolitiques. Certes, ils ne se privaient pas pour condamner la politique du régime et accuser les bolcheviks d'avoir confisqué les acquis révolutionnaires des masses. Mais jamais ils n'eurent l'idée de mettre sur pied une forme de contre-pouvoir. On se demande si un révolutionnaire qui se respecte les reconnaîtrait comme compagnons d'armes.

Que faire donc des contestataires d'Ivanovo ? Ou encore, pour poser la question dans les termes de la nouvelle orthodoxie, comment caractériser leur identité ? Il est évident qu'ils n'avaient rien de sujets libéraux. De plus, la représentation que les révoltés se faisaient d'eux-mêmes en tant qu'héritiers de la Révolution et porte-parole de la classe ouvrière atteste qu'ils restaient prisonniers des valeurs soviétiques. La cause est donc entendue: les ouvriers rebelles n'étaient pas à même de s'imaginer comme des personnalités autonomes, ils ne pouvaient pas s'exprimer comme des sujets libres. Partant, ils étaient incapables de résister au régime.

Affaire classée. Mais encore faudrait-il arriver à déterminer si l'homme est à même de s'affranchir entièrement de son milieu. On peut même émettre l'hypothèse que plus il s'y efforce, plus il entre en interaction avec son environnement, de sorte qu'il devient partie prenante d'un contexte qui inclut ce milieu qu'il veutrejeter.

Le raisonnement n'est pas totalement étranger aux tenants d'une résistance impossible. Dès lors, il est probable qu'ils attendent trop des ouvriers d'Ivanovo. Et, par voie de conséquence, des humains en général. Si l'autonomie du sujet est réellement indispensable pour être à même de résister, on peut arrêter tout de suite de travailler sur le concept de résistance. C'est un peu ce que l'orthodoxie actuelle de la recherche sur l'URSS propose. 
17 Mais que se passe-t-il si la résistance ne relève pas de la construction d'une identité qui se veut indépendante? Y a-t-il des garanties que les affinités avec un système social n'excluent pas que l'on conteste ses pratiques? Est-il inimaginable que les normes que l'on intériorise soient parfois hétérogènes, voire contradictoires? Est-il impossible que le même individu et le même groupe s'identifient à plusieurs rôles qui ne s'accordent pas forcément? Est-il impensable que le concept d'identité, qui postule une certaine stabilité du moi, ne saisisse pas nécessairement les dissonances, les incertitudes, les tâtonnements et les égarements de la personne, et surtout les changements radicaux dans ses engagements? Des êtres captifs ne sauraient-ils tendre vers l'émancipation, tout en sachant (ou non) que leurs efforts sont voués à l'échec?

18 Soit autant de questions rendues plus ardues par cette fixation sur l'identité. Celle-ci n'interdit pas complètement d'en poser quelques-unes. Mais elle propose des réponses toutes faites qui sont en lien avec l'intégration inévitable du sujet dans un réseau remarquablement homogène de normes bolcheviques et avec l'attraction irrésistible du projet soviétique.

19 Le lecteur attentif de Rossman atterrit dans une URSS sans sujet générique. L'auteur ne résiste pas aux chants des sirènes, si bien que ce thème de l'identité ouvrière s'invite forcément dans l'ouvrage. Mais il s'agit d'un moi tellement fragmenté, déséquilibré, mouvant et chargé des sédiments de représentations collectives hétéroclites qu'on a du mal à s'y retrouver. Quelle peut être la plus-value heuristique du concept d'identité s'il est tellement dilué qu'il recouvre tout ce qui motive des masses manifestement hétérogènes? Tout porte à penser que l'hypothèse d'un moi ouvrier est une abstraction quelque peu émoussée qui ne dit pas grand-chose des personnes ni des engagements dans le concret.

20 L'instant saisi par Rossman est un moment privilégié où les insurgés furent acculés à rejeter tout ce le régime représentait. Moment rare où il leur fallut choisir entre deux alternatives au sens original $\mathrm{du}$ terme, c'est-à-dire entre deux possibilités contradictoires. Il ne s'agissait rien de moins que de s'appuyer sur un point d'Archimède, sur l'utopie au sens étymologique du mot : sur un $u$-topos, un lieu imaginable au-delà de tout univers donné et plausible. Il est indifférent que les bolcheviks se soient réclamés de ce lieu. Ce qui compte, c'est que les ouvriers mutins les aient traités d'imposteurs, avec toutes les raisons de le faire. Rossman décrit en détail l'histoire de Kapiton Klepikov, l'un des meneurs de la grève de 1928, qui n'a pas renoncé à ses idées, même entre les mains de la police secrète. Le projet soviétique qu'il chérissait dans son for intérieur avait manifestement peu de choses en commun avec celui des bolcheviks. Quel nom donner à son entêtement? Il a indéniablement rejeté le régime à partir de positions qui étaient diamétralement opposées à celles de l'État-parti. Pourquoi refuser de le désigner comme résistant?

21 Le régime a indiscutablement réussi à faire passer son paternalisme pour une forme d'économie morale. C'est comme celaqu'il est parvenu à se justifier, à l'occasion même devant des gens qu'il maltraitait. Mais rien ne laisse croire que tout le monde était consentant. L'économie morale à la bolchevik avait de faibles chances de succès auprès de ceux qui continuaient à chérir l'imagerie traditionnelle, parmi les nostalgiques de l'ancien régime, chez ceux qui pensaient que le meilleur des mondes possibles se trouvait à l'étranger et ceux qui étaient convaincus que l'État-parti avait trahi le bolchevisme authentique. 

un monde au-delà du pays des soviets. Elle interdit de voir l'univers disparate des sphères culturelles et les interprétations, souvent contradictoires, qu'elles autorisent pour les acteurs historiques, en particulier s'ils nourrissent des projets messianiques aux racines hétéroclites. Ce postulat est tributaire d'une anthropologie unidimensionnelle qui ignore le syncrétisme des visions humaines. Il implique que, nonobstant quelques doutes, l'homo sovieticus ait conservé un primum movens dans les profondeurs de sa personnalité qui n'aurait été, somme toute, que la grande promesse proposée par l'Étatparti.

27 Les hypothèses sur l'identité et le sujet se confondent dans la recherche sur l'Union soviétique. Elles restent désarmées devant la capacité de l'homme à affronter les conditions qui le reproduisent au nom d'imageries utopiques qu'il croit inhérentes au monde qu'il rejette. La thèse d'une résistance inconcevable et d'une subjectivité à jamais assujettie ne relancerait-elle pas le vieux débat sur le pouvoir absolu en URSS, sur son emprise sur les individus, sur les fissures qu'ouvraient les tentatives mêmes de le mettre en œuvre et sur les idées qui motivaient les puissants et les faibles? Rossman rouvre le dossier et il a bien raison. 


\section{NOTES}

1. Pour quelques textes fondateurs de cette tendance, voir Jochen Hellbeck, "Speaking Out: Languages of Affirmation and Dissent in Stalinist Russia ", Kritika, 1, Winter 2000, p. 71-96; Anna Krylova, "The Tenacious Liberal Subject in Soviet Studies ", ibid., p. 119-146; Michael David-Fox, « Whither Resistance ?», ibid., p. 161-165. 